\title{
Brain oscillatory complexity across the life span
}

Alberto Fernández, ${ }^{\mathrm{a}, \mathrm{b}}$ Pilar Zuluaga, ${ }^{\mathrm{c}}$ Daniel Abásolo, ${ }^{\mathrm{d}}$ Carlos Gómez, ${ }^{\mathrm{e}}$ Anahi Serra, ${ }^{\mathrm{a}}$ María Andreina Méndez, ${ }^{\mathrm{f}}$ Roberto Hornero ${ }^{\mathrm{e}}$

åDepartamento de Psiquiatría y Psicología Médica, Facultad de Medicina, Universidad Complutense de Madrid, Madrid, Spain

${ }^{\mathrm{b}}$ Laboratorio de Neurociencia Cognitiva y Computacional. Centro de Tecnología Biomédica, Universidad Politécnica de Madrid y Universidad Complutense de Madrid, Madrid, Spain

${ }^{c}$ Departamento de Estadística e Investigación Operativa, Facultad de Medicina, Universidad Complutense de Madrid, Madrid, Spain

${ }^{\mathrm{d}}$ Centre for Biomedical Engineering, Division of Mechanical, Medical \& Aerospace (MMA) Engineering, Faculty of Engineering and Physical Sciences (J5), University of Surrey, Guildford, UK

${ }^{\mathrm{e}}$ Grupo de Ingeniería Biomédica. Universidad de Valladolid, Spain.

f South West London and St George's Mental Health Trust, UK

\section{Corresponding author:}

Alberto Fernández

Departamento de Psiquiatría y Psicología Médica

Facultad de Medicina

Universidad Complutense de Madrid

Avda. Complutense s/n 28040

Madrid 
Spain

Tel: +34913942296

Fax: +34913941503

E-mail: aferlucas@med.ucm.es

\section{Abstract}

Objective: Considering the increasing use of complexity estimates in neuropsychiatric populations, a normative study is critical to define the 'normal' behaviour of brain oscillatory complexity across the life span.

Method: This study examines changes in resting-state magnetoencephalogram (MEG) complexity - quantified with the Lempel-Ziv complexity (LZC) algorithm - due to age and gender in a large sample of 222 (100 males/122 females) healthy participants with ages ranging from 7 to 84 years.

Results: A significant quadratic (curvilinear) relationship $(p<0.05)$ between age and complexity was found, with LZC maxima being reached by the sixth decade of life. Once that peak was crossed, complexity values slowly decreased until late senescence. Females exhibited higher LZC values than males, with significant differences in the anterior, central and posterior regions $(p<0.05)$.

Conclusions: These results suggest that the evolution of brain oscillatory complexity across the life span might be considered a new illustration of a 'normal' physiological rhythm.

Significance: Previous and forthcoming clinical studies using complexity estimates might be interpreted from a more complete and dynamical perspective. Pathologies not 
only cause an 'abnormal' increase or decrease of complexity values but they actually 'break' the 'normal' pattern of oscillatory complexity evolution as a function of age.

Keywords: Life Span, Ageing, Complexity, Brain Development, White Matter Development

\section{Highlights}

1. A significant quadratic (curvilinear) relationship between age and oscillatory complexity exists, with complexity maxima reached by the sixth decade of life.

2. As in previous studies, females exhibit higher complexity values than males, at least in some brain regions.

3. The evolution of oscillatory complexity across the life span is interpreted as a physiological rhythm which is altered by several brain pathologies.

\section{INTRODUCTION}

Neurophysiological studies of human brain have emphasised the critical role of age effects in the electroencephalograms (EEGs) or magnetoencephalograms (MEGs) of healthy individuals. As Clarke et al. (2001) pointed out, EEG maturational changes were reported even in very early investigations (Lindsley 1939). Matousek and Petersén 
(1973) established some 'norms' for the developing EEG in a large sample of healthy individuals aged 1-21 years. John et al. (1980) further studied the developmental aspects of the EEG in healthy children and calculated a series of 'developmental equations' that demonstrated a linear behaviour of the conventional delta, theta, alpha and beta bands. Low-frequency bands exhibited a negative slope as a function of age while high-frequency bands exhibited the opposite tendency. In essence, a 'substitution process' occurs. Low-frequency bands (delta and theta) are predominant until the age of 4 years, but both show a sustained decrease. As age increases, the dominant lowfrequency bands are substituted by activity in the alpha and beta frequency ranges. Thus, the mean frequency of the so-called 'central alpha' is $7 \mathrm{~Hz}$ by the first year of life, $9 \mathrm{~Hz}$ by 4 years of age and stabilises at around $10 \mathrm{~Hz}$ in mild adolescence (see Marshall et al., 2002). Alpha rhythm in the $8-12 \mathrm{~Hz}$ frequency band becomes the most prominent rhythm in the awake EEG and MEG of healthy adults.

Adolescence is a key transition point for the oscillatory activity in the brain. During adolescence, a significant tendency to reduced power in all frequency bands was observed (Gasser et al., 1988); a power reduction that correlated with a decrease of grey-matter volumes in the transition from infancy to early adolescence (Whitford et al., 2007). These observations are supported by Dustman et al. (1999); they confirmed that such decrease of absolute power continues into adulthood, although changes are not so radical when compared to the transition between infancy and adolescence. Finally, healthy ageing is defined by a new 'substitution process' in the spectral profile, characterised now by the so-called 'slowing' of EEG and MEG traces. Overall, a pronounced decrease in the amplitude of the basic alpha rhythm $(8-12 \mathrm{~Hz})$ has been noticed, accompanied by a power increase in the theta and delta frequency ranges. Interestingly, during this new substitution process, low-frequency bands also increase 
their topographic location, following a posterior-to-anterior tendency (see John et al., 1988, and the review by Rossini et al., 2007).

All the above-mentioned studies used classical spectral analysis methods to investigate developmental changes. Traditional methods have been challenged by new techniques derived from the non-linear analysis theory, since EEG and MEG signals can be regarded, at least to some extent, as generated by complex systems with non-linear dynamics (Lopes da Silva, 1991; for a critical review on this issue see also Stam, 2005). Complexity analysis is a particular form of non-linear analysis that has been applied to EEG or MEG data. Unfortunately, there is no consensus for a unique definition of the term complexity within this background, and several estimates have been proposed. For example, Tononi and co-workers' (1994) proposed a measure, called 'neural complexity $(\mathrm{CN})^{\prime}$, which can be defined as a balance between functional segregation and integration in the brain. The correlation dimension is a widely used method that seems to represent a non-linear estimate of the number of independent neuronal populations or oscillators which give rise to an EEG/MEG signal (Lutzenberger et al., 1995). The algorithmic complexity (Kolmogorov, 1965) is defined as the length of the shortest computer programme that generates a particular bit string. Most of these complexity estimates might be interpreted as a measure of the regularity/variability of brain oscillations and/or an attempt to evaluate the number of independent oscillators or frequency components underlying the observed signal (Aboy et al., 2006; Lutzenberger et al., 1995).

Complexity estimates have been specifically employed to investigate developmental changes of brain oscillatory activity measured with EEG. Anokhin's group (Anokhin et al., 1996) performed dimensional complexity analysis in a large sample of healthy males with an age range from 7 to 60 years. Complexity values increased monotonously 
as a function of age. Gender effects on maturational changes were not investigated in these first studies. In an ulterior investigation, Anokhin's group analysed dimensional complexity values in a new sample, now including healthy females with an age range from 7 to 66 years (Anokhin et al., 2000). Age effects were identical to those observed in their previous study. However, gender emerged as an important variable, since results indicated higher complexity values in females. Girls exhibited higher complexity values when compared to boys, and gender differences increased until adolescence. The authors interpreted these findings as an evidence of faster maturation of cortical activity in females. In parallel, Meyer-Lindenberg (1996) confirmed Anokhin's results using correlation dimension $\left(D_{2}\right)$ and the first Lyapunov exponent $(L 1)$. It is noteworthy that gender differences were also reported in some developmental EEG studies using conventional spectral measures (see, e.g., Clarke et al., 2001).

Age-related changes of brain signals have been also investigated by means of estimators such as sample entropy and multi-scale entropy. For example, multi-scale entropy values were calculated by McIntosh et al. (2008) to assess age-related trial-to-trial variability in a face-recognition visual memory task. Results indicated that brain signal variability increased with age, and showed a positive correlation with subjects' accuracy on task performance. Authors understood that brain maturation increases brain signal variability and this process is accompanied by an increase in behavioural stability. Lippé at al. (2009) calculated multi-scale entropies of visual and auditory-evoked responses in a sample of healthy infants and children aged 1 month-5 years of age. As in all previous studies, complexity increased with age, although signal complexity was higher for visual as compared to auditory stimuli. Bruce et al. (2009) accomplished an interesting study where the regularity of EEG signals during sleep was compared in samples of middle-aged and elderly individuals. Signal complexity was estimated by 
means of sample entropy, and results indicated that sample entropy was larger in elderly individuals in sleep stage 2 .

Overall, these investigations basically support the notion of an uninterrupted, linear increase of brain oscillatory complexity during maturation and ageing. In a recent MEG study (Fernández et al., 2010), we suggested that such uninterrupted complexity increase observed in Anokhin's studies may be explained by the characteristics of the sample. Our sample was composed of subjects between the sixth and eighth decades of life, and a linear decrease of complexity scores as a function of age was observed. These apparent contradictions suggest that normative studies with larger samples and more robust complexity estimates are needed.

Previous investigations used methods derived from the chaos theory, such as $D_{2}$ and $L 1$. The use of these estimates to characterise biomedical time series poses significant problems. First, to accurately compute both metrics, one needs an amount of data beyond the experimental possibilities for biomedical time series (Eckmann \& Ruelle, 1992). In addition, time series need to be stationary, something that is usually not true with physiological signals. With these limitations in mind, Lempel-Ziv Complexity (LZC), a complexity estimator introduced by Lempel and Ziv (1976), has been proposed for EEG/MEG signals analysis. The LZC is a metric that, similar to the algorithmic complexity, reflects the number of distinct substrings and the rate of their recurrence along the given sequence (Radhakrishnan \& Gangadhar, 1998). Larger LZC values correspond to more complex time series. One important advantage of this metric is that it can be calculated even for short data segments and in non-stationary signals (Zhang et al., 1999). Moreover, LZC is more precise than $L 1$ for characterising order or disorder (Kaspar \& Schuster, 1987) and is better suited for the electromagnetic brain activity analysis than $D_{2}$ (Zhang et al., 2001). 
LZC has been used to analyse EEG and MEG signals in patients with Alzheimer's disease (Abásolo et al., 2006; Fernández et al., 2010; Gómez et al., 2006), attention deficit-hyperactivity disorder (ADHD) (Fernández et al., 2009), depression and schizophrenia (Li et al., 2008; Fernández et al., 2011a; Méndez et al., 2011;) as well as to measure the depth of anaesthesia (Zhang et al., 2001), or to study epileptic seizures (Radhakrishnan and Gangadhar, 1998). The increasing clinical use of LZC and other estimates of oscillatory complexity is the main reason to carry out a normative study where the 'normal' behaviour of complexity values is defined according to age and gender influences in a large population. This is the main goal of our investigation.

\section{METHODS}

\subsection{Participants}

Our sample consisted of 222 (100 males/122 females) healthy right-handed participants. Subjects' age ranged from 7 to 84 years (mean age \pm standard deviation: $43.83 \pm 21.62$ years). No significant differences in terms of age were found between males (42.26 \pm 21.08) and females $(45.11 \pm 22.05)$. All participants included in the current investigation have been described in some of our previous studies (Fernández et al., 2002; Fernández et al., 2009; Fernández et al., 2010; Fernández et al., 2011a; Méndez et al., 2011; Solesio et al., 2009).

\subsection{MEG Data Collection}

MEGs were acquired with a 148-channel whole-head magnetometer (MAGNES 2500 $\mathrm{WH}^{\circledR}, 4 \mathrm{D}$ Neuroimaging, San Diego, CA, USA) placed in a magnetically shielded room at 'Centro de Magnetoencefalografía Dr. Pérez-Modrego' (Madrid, Spain). Subjects were in an awake but resting state with their eyes closed and under vigilance control 
during the recording. They were asked to avoid blinking and making movements. For each subject, 5 min of MEG signal were acquired at a sampling frequency of $678.17 \mathrm{~Hz}$ using a hardware band-pass filter of $0.1-200 \mathrm{~Hz}$. Afterwards, these recordings were downsampled by a factor of $4(169.549 \mathrm{~Hz}, 50863$ samples $)$. This process consisted of filtering the data to avoid aliasing (Nyquist criterion) and downsampling the recordings. The anti-aliasing filter was a second-order Butterworth IIR routine applied to the signals in both forward and reverse directions to avoid net phase shift with cut-off frequency at $76.30 \mathrm{~Hz}(45 \%$ of the final sample rate: $169.549 \mathrm{~Hz})$.

Artefact-free epochs of $20 \mathrm{~s}$ were selected off-line. Finally, these epochs were processed using a band-pass filter with Hamming window and cut-off frequencies at 1.5 and 40 Hz. This filter was used to remove the power line frequency (50 Hz in Europe) and the DC component from the MEG data.

\subsection{LZC calculation}

LZC is a non-parametric measure for finite sequences related to the number of distinct substrings and the rate of their occurrence along the sequence, with larger values corresponding to more complexity in the data (Zhang et al., 2001). LZC analysis is based on a coarse-graining of the measurements, so the MEG recording must be first transformed into a finite symbol string. In this study, a binary sequence conversion was used. By comparison with a threshold $T_{d}$, the original data are converted into a $0-1$ sequence. We used the median as the threshold $T_{d}$ due to its well-known robustness to outliers (Nagarajan et al., 2002). The binary string obtained is then scanned from left to right and a complexity counter $c(n)$ is increased by one unit every time a new subsequence of consecutive characters is encountered in the scanning process. To obtain a complexity measure which is independent of the sequence length $n, c(n)$ should be normalised. In general, $b(n)=n / \log _{2}(n)$ is the upper bound of $c(n)$ for a binary 
sequence (Zhang et al., 2001). Thus, $c(n)$ can be normalised via $b(n): C(n)=c(n) / b(n)$. The normalised LZC, $C(n)$, reflects the arising rate of new patterns along with the sequence.

The detailed algorithm for the measure of the LZC is included in Appendix A.

\subsection{MEG Data Reduction and Analysis}

A normalised LZC value was obtained for each channel and participant. Thus, statistical analyses were performed with 148 LZC scores per subject. As in previous studies (Fernández et al., 2009; Fernández et al., 2010; Fernández et al., 2011a; Méndez et al., 2011), the initial 148 LZC values were averaged into five regions: anterior, central, left lateral, right lateral and posterior, which are included as default sensor groups in the 4Dneuroimaging source analysis software (see Fig. 1).

\subsection{Statistical Analysis}

The statistical analyses were performed by using Excel, Statistical Package for the Social Sciences (SPSS) 19 and Statgraphics 5.1 software. Results are presented as mean \pm standard error. Probabilities $p<0.05$ were considered as significant. We studied the effect of age and gender on LZC variables by means of two-way analysis of variance (ANOVA).The relationship between LZC scores and age was determined by means of linear and polynomial regression models. Finally, we examined regional differences among LZC variables by means of one-way repeated measures ANOVA with a covariate (age) and a between-groups factor (gender). Bonferroni correction was used for multiple comparison tests.

\section{RESULTS}

\subsection{Age and Gender Effects on LZC}


In previous studies, we found that age and gender might be modifying factors of LZC values (Fernández et al., 2011a; Méndez et al., 2011). Thus, our study began with an exploratory analysis by describing data according to these two variables. To this aim, participants were subdivided into six age groups: below 19 years $(<19), 19-40,41-60$, 61-70 and above 70 years (>70) (see Fig. 2). Age effects were significant for all LZC variables (all $p$ values $<0.001$ ). Gender had a statistically significant effect in central ( $p$ $=0.006)$ and posterior $(p=0.010)$ LZC indicating that, overall, females showed higher values in these regions. However, the age $\times$ gender interaction was only significant for the anterior region $(p=0.007)$. When this interaction was further explored by means of pairwise comparisons using Bonferroni correction, we concluded that females' LZC values were higher than those observed in males but this effect was only significant for individuals below 19 years $(p=0.007)$. To better analyse this effect, we investigated anterior LZC values within the $<19$ years group. Thus, separated linear regression models were fitted for males and females, considering age as an independent variable. The slopes of both models were significantly different from 'zero' (slope $=0.014 ; p=$ 0.0415 ) for males and (slope $=0.0053 ; p=0.0322$ ) for females, indicating a positive (i.e., increasing) tendency of LZC values in both gender groups. Moreover, the slope of the regression line was significantly larger in males than in females $(p=0.045)$, representing a steeper increase of anterior LZC scores in the males group. The intercept term $\left(b_{0}=0.588\right.$ for females and 0.464 for males) was larger in females than in males ( $p$ $=0.048$ ), indicating that anterior LZC values were significantly higher in females within the $<19$ years group.

Once the exploratory analysis was concluded, the next goal was to model the age influence on MEG signals by means of LZC. Intuitively, looking at Figure 3, it might be 
observed that LZC values seem to increase till they reach a certain maximum, and after that point they start to decrease. This suggests to fit a quadratic function $\left(b_{0}+b_{1}\right.$ age + $\mathrm{b}_{2} * \mathrm{age}^{2}$ ) to model such behaviour. Fittings of the quadratic function are displayed in Tables 1 and 2 . Here, it is important to note that polynomial regression models were calculated separately for males and females in anterior, central and posterior regions, since gender exerted a significant influence on complexity values within these regions. A single model (females + males) was calculated for left and right lateral, since gender had no effect on the LZC values of these regions.

Results demonstrated that all the $\mathrm{b}_{2}$ coefficients were significant (all $p$ values $<0.0467$ ), confirming the adequacy of a quadratic rather than a linear model to explain age effects in all regions (see Fig. 4). This means that, as intuitively noticed, LZC values increase (with a brisker increase from infancy to adolescence-early adulthood) till they reach a maximum at a certain age and then they tend to slowly decrease. Since results confirmed the adequacy of a quadratic model, maxima were calculated for each region using the formula:

Maximum $=-\frac{b_{1}}{2 * b_{2}}$

As it can be observed in Table 2, the complexity maximum in the anterior region was reached at a younger age than in other regions, that is, the complexity values started to decline earlier. Furthermore, this decline occurred before in females (51.09 years) than in males (64.03 years). A similar tendency was observed in the central region, whereas we found the opposite trend in the posterior region. Of note, the age $\times$ gender interaction previously detected in anterior LZC scores also had some influence on this modelling. As displayed in Figure 3, males' and females' regression curves reach an intersection point. Such an intersection point occurs at the age of 66.54 years. 
Previously, we claimed that females (especially the youngest sample) showed overall higher LZC values within this region, but this affirmation is only true until this intersection point (i.e., until the age of 66.54 years). Once this point is crossed, the decline of LZC is more pronounced in females, and as a consequence males exhibit higher LZC values.

\subsection{LZC differences across regions}

Considering that previous studies reported regional differences in complexity values, we investigated this potential effect in our sample. Results showed a significant effect of region $(p<0.001)$ and age $(p<0.001)$. As we have described (see subsection 3.1.), there is a general tendency to increased LZC values as a function of age in the five LZC regions. However, LZC values were clearly different when regional effect was considered (see Fig. 2). Bonferroni pairwise comparisons showed that anterior and central LZC values were significantly higher when compared to left and right lateral or posterior regions (all $p$ values $<0.001$ ). Gender effect was very close to the level of statistical significance $(p=0.053)$. Overall, LZC scores in anterior and central regions were significantly higher in both males and females, but a slightly different behaviour was found in the anterior region. As we previously described, anterior region LZC values for older females $(>70)$ tend to show a more pronounced decline when compared to males' values. As a consequence, anterior LZC values in the older females are closer to the values of left lateral, right lateral and posterior regions than in the males group.

\section{DISCUSSION}

These results represent the first normative study where complexity values have been obtained in such a large sample of healthy individuals between the first and eighth 
decades of life. When compared with previous investigations (Anokhin et al., 1996, Anokhin et al., 2000; Meyer-Lindenberg 1996, McIntosh et al., 2008; Pravitha et al., 2005), three major coincidences were observed: (1) complexity values tended to increase as a function of age, (2) although such increase was present in all brain regions, complexity values in anterior and central regions were significantly higher compared with other regions (see Fig. 4), probably mirroring the greater functional and anatomical intricacy of frontal lobes (Fuster, 2002) and (3) females exhibited higher complexity values than males, although in our study this effect was only significant in anterior, central and posterior regions. When healthy subjects in their seventh and eighth decades of life were included in the sample, a significant quadratic, rather than linear, relationship between age and complexity was detected. A quadratic relationship meant that complexity values tended to increase until they reached a maximum or peak. The age of peak was variable and particular for each brain region but, interestingly, it basically coincides with the upper limit of Anokhin's group samples (Anokhin et al., 1996; Anokhin et al., 2000) where linear behaviours were described. This is to say that, in our sample, complexity peaks were reached by the sixth decade of life in most brain regions. Once that peak was crossed, complexity values slowly decreased until late senescence.

The first issue addressed by these evidences is the relationship between the evolution of conventional spectral parameters and the evolution of complexity scores. The evolution of EEG frequency bands as a function of age in children and young adults is wellknown (Matousek and Petersén, 1973; John et al., 1980). Subsequent investigations (John et al., 1988) allowed the generalisation of the so-called 'developmental equations' in middle-aged and elderly individuals. More importantly, the spectral evolution with age has been previously compared with the evolution of complexity values. Anokhin 
and co-workers (1996) found that a significant decrease of theta and alpha power was observed during maturation, with only slight changes during middle age. On the contrary, complexity scores showed a linear increase, with an abrupt augmentation from infancy to adolescence (especially in frontal regions), and a sustained augmentation (uniform across brain regions) from adolescence to adulthood. This seminal study suggested that spectral and complexity analyses offer two different and complementary perspectives on brain maturation. The neurobiological basis of the divergence between spectral and complexity evolutions with age have been recently established. Whitford et al. (2007) investigated the correlation between grey matter (GM), white matter (WM) and relative power in the conventional frequency bands. They hypothesised that the significant reduction of GM volume observed in the transition from infancy to adolescence should produce a reduction of the EEG power, and results confirmed their hypothesis. The linear reduction of GM volume was associated with a reduction of relative power, especially in the low-frequency bands. No correlations were found between WM volume and relative power in any band. Very recently (Fernández et al., 2011b), we demonstrated that WM integrity is strongly correlated with MEG complexity, as measured by LZC. Consequently, we claimed that our own findings and Whitford and co-workers' results opened the window to the perspective of two physiologically independent processes regulating the properties of EEG/MEG signals: those associated with GM (conventional linear spectral measures) and those associated with WM (non-linear complexity estimators).

Considering these facts, our results pose two new critical questions: (1) how can we explain such a quadratic relationship between age and oscillatory complexity? and (2) is there any physiological process in the brain with a similar behaviour? If we accept that complexity values estimate the number of independent oscillators (Lutzenberger et al., 
1995) and/or the number of frequency components that compose the observed signals (Aboy et al., 2006), then we should hypothesise that the underlying physiological process must be closely related to the generation of oscillatory activity in the brain. Anokhin's group proposed that the observed linear increase of complexity values as a function of age might be explained by the "continuous formation and modification of neural cell assemblies' that relies on the 'developmental selection of cortico-cortical connections favouring a synchronous excitation of distributed neurons' (Anokhin et al., 1996). Following these authors' reasoning, the proposed underlying process for an uninterrupted complexity increase should continue until, at least, middle age. An excellent candidate to elucidate this process is the myelination of cortical WM, which is intimately involved in the formation of cortico-cortical connections.

The myelination of cortical WM might allow explaining a process that exceeds the period of brain maturation but, according to current knowledge, it would not explicate a sustained linear increase of complexity values. Some classical studies (Yakovlev \& Lecours, 1967) reported a protracted cycle of myelination that continued into the third decade of life. However, cortical WM development with age seems to follow a quadratic rather than linear behaviour. This effect was first described in some volumetric studies (Bartzokis et al., 2001; Sowell et al., 2003; Sowell et al., 2004) where WM volumes increased until reaching a certain peak, frequently in the fourth decade of life, and then decreased until senescence. The quadratic relationship between age and WM has been confirmed by more recent studies using fractional anisotropy (FA). The FA is a diffusion tensor imaging (DTI)-derived measure that is particularly useful as an estimate of the microstructure and specific organisation of myelinated axonal fibres, and may be considered an estimate of WM integrity (Basser \& Pierpaoli, 1996). Using this measure, several authors (Hasan et al., 2007, Hasan et al., 2009; 
Kochunov et al., 2012; McLaughlin et al., 2007) reported quadratic effects of age on FA values, with ages of peak between the third and fifth decades of life and a very slow decrease of FA values from these peaks to late senescence. Consequently, we can affirm that the quadratic relationship between age and oscillatory complexity shown by our results parallels the quadratic relationship between age and cortical WM.

The similarity of global age-related trajectories is not the only coincidence between complexity and WM. One of our findings, which replicated previous studies, was the more abrupt increase of complexity values from infancy to adolescence and young adulthood, especially in anterior brain regions. A similar, steeper increase of FA values from infancy to adolescence has been found in some studies (Lebel et al., 2008; McGraw et al., 2002; Snook et al., 2005). Also, our results showed that complexity peaks were reached at an earlier age in anterior brain regions, and therefore complexity decline started earlier there. Comparably, Bartzokis et al. (2001) described earlier peaks of WM volume in frontal regions, while Nusbaum et al. (2001) and Pfefferbaum et al. (2005) supported this tendency in DTI investigations.

At this point, it might be argued that all those similarities between complexity and WM are interesting but probably too incidental to establish an actual association. As mentioned above, in a previous study, we studied the relationship between LZC and WM. Although this in vivo study proved a positive correlation between WM integrity (i.e., FA values) and complexity, it might be still questioned if such association meets the rationale of our previous hypothesis, that is, if WM is related to the generation of oscillatory activity in the brain. EEG/MEG signals are composed of the summation of multiple electromagnetic oscillations at different frequencies which derive from the collective and synchronous behaviour of neural populations located in different brain regions (Cantero et al., 2009; Segalowitz et al., 2010). Therefore, synchronisation 
among brain regions appears as a basic mechanism to explain the frequencies' variability observed in EEG/MEG signals, which is in turn intimately related to oscillatory complexity estimates (Lutzenberger et al., 1995). Is WM involved in synchronisation? The excellent review by Fields (2008) might answer this question. Fields (2008) points out that WM has been traditionally considered a passive insulation substance that ensures the transmission of neural impulses. The author proposes that emerging evidence indicates this is a too simplistic perspective, and WM also participates in the speed control of impulse conduction and consequently in the synchronisation among cortical regions.

Nevertheless, the assumed relationship between WM and oscillatory complexity cannot fully explain all the findings in our study. For example, the complexity peaks are found at older ages than the peaks observed in WM studies. Such a delay may indicate that a supplementary and currently unknown process is postponing complexity decrease in aged individuals. Here, it is important to note that the 'ages of peak' in WM investigations are variable and, in some particular cases, the decline of FA scores in aged subjects was not significant (McLaughlin et al., 2007). Moreover, some classical studies (Benes et al., 1994; Pfefferbaum et al., 1994) reported constant WM volumes or even signs of myelination until the seventh decade of life. Yet, we still claim that some additional processes or confounding factors must be investigated in the future. Similarly, females exhibited higher complexity values than males and, to the best of our knowledge, no clear WM-related differences between genders have been reported that might explain this effect. Although there is no straightforward explanation for a greater complexity in females' brain signals, this finding appeared in all studies investigating gender influences on complexity values (Anokhin et al., 2000; Pravitha et al., 2005). Unfortunately, the finding was scarcely discussed in physiological terms. Gender 
differences in brain weight and structure have been reported in several studies during the last decades (Dekaban, 1978; Peters, 1991; Witelson, 1989; Witelson et al. 1995), but the implication of such differences in complexity values is difficult to determine. The study that might be more closely related is that by Benes et al. (1994), where significantly higher myelination ratios were reported in females until young adulthood. Interestingly, a recent investigation (Luders et al., 2004) informed of a higher 'cortical complexity' in females, with cortical complexity defined as a gyrification measure which estimates the frequency of sulcal and gyral convolutions in some cortical areas. Females exhibited significantly greater cortical complexity scores in most areas, and authors interpreted this finding to be associated with the underlying cytoarchitecture and with specific connectivity patterns in the brain. The relationship between cortical complexity and EEG/MEG complexity estimates should also be investigated in the future.

Finally, very recent studies have investigated how the functional organisation of the brain may affect the complexity of brain signals. For example, Vakorin et al. (2011) explored the relationship between the complexity of individual sources within a network and the information exchange between them. Results indicated that the amount of information transferred from one source to another correlated with differences in the complexity (as estimated with sample entropy) of those sources. Authors interpreted their findings by claiming that the propagation of information within a network may be described as an accumulation of complexity of the brain signals. Misic et al. (2011) investigated the relationship between the variability of a region's activity and the topological role of that region in a functional network. They recorded resting-state EEGs and constructed graphs of functional networks. Some graph measures such as centrality, efficiency and 'betweenness' were estimated to analyse the network 
behaviour. Their results showed that the centrality of network nodes (i.e., nodes with higher number of connections) predict the information content of their physiological activity. This might be related with the evidence of a more robust developmental increase of entropy values in brain areas such as the precuneus and the posterior cingulate. These areas are characterised by their high extrinsic and intrinsic connectivity, and are critical for the default-network model of resting-state activity. Misic et al.'s study may be considered a new confirmation of the positive correlation between complexity and functional connectivity (see Fernández et al., 2011b).

\section{SIGNIFICANCE}

Our study is limited by its cross-sectional nature that extrapolates life span data across multiple cohorts. A longitudinal rather than cross-sectional study would better address the problem of oscillatory complexity evolution across the life span. In spite of this limitation, we still claim that this is a comprehensive investigation performed to understand complexity evolution and its potential physiological determinants. From our point of view, the clinical implications might be of particular relevance. First, normative data derived from a considerably large sample of both genders are now available to compare with pathological populations. Second, and more important in our opinion, previous and forthcoming clinical studies using complexity estimates might be interpreted from a more complete and dynamical perspective (see McKey \& Milton, 1987). Considering preceding investigations, clinical populations of different pathologies exhibited significantly higher or lower complexity values than controls, but such statistical difference only reveals a percentage of the actual dissimilarity between the pathological and the healthy states. Recent studies proved that pathologies such us mild cognitive impairment, depression, schizophrenia or ADHD (see Fernández et al., 
2009; Fernández et al., 2010; Fernández et al., 2011a; Méndez et al., 2011) not only cause an 'abnormal' increase or decrease of oscillatory complexity values but also actually 'break' the 'normal' pattern of complexity evolution as a function of age. For instance, patients with major depression presented higher complexity values than controls, and these values failed to show the 'normal' increase with age that would be expected within the age range of the sample. When patients' symptoms remitted, their complexity values decreased (becoming closer to those of controls) and recovered the tendency to increase as a function of age observed in healthy individuals within the same age range (Méndez et al., 2011). This implies that the disease not only modifies the values of a potential biological marker but, more importantly, alters a physiological rhythm in the organism. In the study presented here, we have described what might be considered a new illustration of a physiological rhythm, that is, the evolution of brain oscillatory complexity across the life span. 


\section{Appendix A. Lempel-Ziv complexity algorithm}

The detailed algorithm for the measure of the $L Z$ complexity is as follows (Zhang et al., 2001):

1. Let $S$ and $Q$ denote two subsequences of the original sequence $P$ and $S Q$ be the concatenation of $S$ and $Q$, while $S Q \pi$ is a string derived from $S Q$ after its last character is deleted ( $\pi$ means the operation to delete the last character).

2. Let $v(S Q \pi)$ denote the vocabulary of all different substrings of $S Q \pi$.

3. At the beginning, the complexity counter $c(n)=1, S=s(1), Q=s(2), S Q=s(1)$, $s(2)$ and $S Q \pi=s(1)$.

4. In general, suppose that $S=s(1), s(2), \ldots, s(r), Q=s(r+1)$ and, therefore, $S Q \pi=$ $s(1), s(2), \ldots, s(r)$. If $Q \in v(S Q \pi)$, then $Q$ is a subsequence of $S Q \pi$, not a new sequence.

5. $S$ does not change and renew $Q$ to be $s(r+1), s(r+2)$, then judge if $Q$ belongs to $v(S Q \pi)$ or not.

6. The steps 4 and 5 are repeated until $Q$ does not belong to $v(S Q \pi)$. Now $Q=$ $s(r+1), s(r+2), \ldots, s(r+i)$ is not a subsequence of $S Q \pi=s(1), s(2), \ldots, s(r+i-1)$, so increase the counter by one.

7. Thereafter, $S$ and $Q$ are combined and renewed to be $s(1), s(2), \ldots, s(r+i)$, and $s(r+i+1)$, respectively.

8. Repeat the previous steps until $Q$ is the last character. At this time, the number of different substrings is $c(n)$, the measure of complexity.

To obtain a complexity measure which is independent of the sequence length, $c(n)$ should be normalised. If the length of the sequence is $n$ and the number of different symbols is $\alpha$, it has been proved (Lempel and Ziv, 1976) that the upper bound of $c(n)$ is given by: 
$c(n)<\frac{n}{\left(1-\varepsilon_{n}\right) \log _{\alpha}(n)}$

where $\varepsilon_{n}$ is a small quantity and $\varepsilon_{n} \rightarrow 0(n \rightarrow \infty)$. In general, $n / \log _{\alpha}(n)$ is the upper limit of $c(n)$, where the base of the logarithm is $\alpha$, i.e.

$\lim _{n \rightarrow \infty} c(n)=b(n) \equiv \frac{n}{\log _{\alpha}(n)}$

For a binary conversion $\alpha=2$, and $b(n)$ is given by

$b(n) \equiv \frac{n}{\log _{2}(n)}$

and $c(n)$ can be normalised via $b(n)$ :

$C(n)=\frac{c(n)}{b(n)}$

$C(n)$ is usually a value between zero and one. The normalised LZC reflects the arising rate of new patterns along with the sequence (Zhang et al. 2001). A minimum data length must be considered to ensure that LZC reveals real data features (Yan and Gao, 2004). Since a previous work showed that the LZC values become stable for MEGs longer than 3000 samples (Gómez et al., 2006), an epoch length of 3392 data points (20 s) was used in the current study. 


\section{Acknowledgements}

This work has been partially supported by Ministerio de Economía y Competitividad and FEDER grant TEC 2011-22987. 


\section{References}

Abásolo D, Hornero R, Gómez C, García M, López M. Analysis of EEG background activity in Alzheimer's disease patients with Lempel-Ziv complexity and central tendency measure. Med Eng Phys 2006; 28: 315-22.

Aboy M, Hornero R, Abásolo D, Álvarez D. Interpretation of the Lempel-Ziv complexity measure in the context of biomedical signal analysis. IEEE Trans Biomed Eng 2006; 53: 2282-8.

Anokhin AP, Birbaumer N, Lutzenberger W, Nikolaev A, Vogel F. Age increases brain complexity. Electroencephalogr Clin Neurophysiol 1996; 99: 63-8.

Anokhin AP, Lutzenberger W, Nikolaev A, Birbaumer N. Complexity of electrocortical dynamics in children: developmental aspects. Dev Psychobiol 2000; 36: 9-22.

Bartzokis G, Beckson M, Lu PH, Nuechterlein KH, Edwards N, Mintz J. Age-related changes in frontal and temporal lobe volumes in men: a magnetic resonance imaging study. Arch Gen Psychiatry 2001; 58: 461-5.

Basser PJ, Pierpaoli C. Microstructural and physiological features of tissues elucidated by quantitative-diffusion-tensor MRI. J Magn Reson B 1996; 111: 209-19.

Benes FM, Turtle M, Khan Y, Farol P. Myelination of a key relay zone in the hippocampal formation occurs in the human brain during childhood, adolescence, and adulthood. Arch Gen Psychiatry 1994; 51: 477-84.

Bruce EN, Bruce MC, Vennelaganti S. Sample entropy tracks changes in electroencephalogram power spectrum with sleep state and aging. J Clin Neurophysiol 2009; 26: 257-66. 
Cantero JL, Atienza M, Cruz-Vadell A, Suarez-González A, Gil-Neciga E. Increased synchronization and decreased neural complexity underlie thalamocortical oscillatory dynamics in mild cognitive impairment. Neuroimage 2009; 46: 938-48.

Clarke AR, Barry RJ, McCarthy R, Selikowitz M. Age and sex effects in the EEG: development of the normal child. Clin Neurophysiol 2001;112: 806-14.

Dekaban AS, Changes in brain weights during the span of human life: relation of brain weights to body heights and body weights. Ann Neurol 1978; 4: 345-56.

Dustman RE, Shearer DE, Emmerson RY. Life-span changes in EEG spectral amplitude, amplitude variability and mean frequency. Clin Neurophysiol 1999; 110 : 1399-409.

Eckmann JP, Ruelle D. Fundamental limitations for estimating dimensions and Lyapunov exponents in dynamical systems. Physica D 1992; 56: 185-187. Fernandez A, Maestu F, Amo C, Gil P, Fehr T, Wienbruch C, et al.. Focal temporoparietal slow activity in Alzheimer's disease revealed by magnetoencephalography. Biol Psychiatry 2002; 52: 764-70.

Fernández A, Quintero J, Hornero R, Zuluaga P, Navas M, Gómez C, et al. Complexity analysis of spontaneous brain activity in attention-deficit/hyperactivity disorder: diagnostic implications. Biol Psychiatry 2009; 65: 571-7.

Fernández A, Hornero R, Gómez C, Turrero A, Gil-Gregorio P, Matias-Santos J, et al. Complexity analysis of spontaneous brain activity in Alzheimer disease and mild cognitive impairment: an MEG study. Alzheimer Dis Assoc Disord 2010; 24: 182-9. Fernández A, López-Ibor MI, Turrero A, Santos JM, Morón MD, Hornero R, et al. Lempel-Ziv complexity in schizophrenia: A MEG study. Clin Neurophysiol 2011; 122: $2227-2235$. 
Fernández A, Rios-Lago M, Abásolo D, Hornero R, Álvarez-Linera J, Paul N, et al. The correlation between white-matter microstructure and the complexity of spontaneous brain activity: A difussion tensor imaging-MEG study. Neuroimage 2011; 57: 13001307.

Fields RD. White matter in learning, cognition and psychiatric disorders. Trends Neurosci 2008; 31: 361-70.

Fuster JM. Frontal lobe and cognitive development. J Neurocytol 2002; 31: 373-85. Gasser T, Verleger R, Bacher P, Sroka L. Development of the EEG of school-age children and adolescents. I. Analysis of band power. Electroencephalogr Clin Neurophysiol 1988: 69: 91-9.

Gómez C, Hornero R, Abásolo D, Fernández A, López M. Complexity analysis of the magnetoencephalogram background activity in Alzheimer's disease patients. Med Eng Phys 2006; 28: 851-9.

Hasan KM, Kamali A, Iftikhar A, Kramer LA, Papanicolaou AC, Fletcher JM, et al. Diffusion tensor tractography quantification of the human corpus callosum fiber pathways across the lifespan. Brain Res 2009; 1249: 91-100.

Hasan KM, Sankar A, Halphen C, Kramer LA, Brandt ME, Juranek J, et al. Development and organization of the human brain tissue compartments across the lifespan using diffusion tensor imaging. Neuroreport 2007; 18: 1735-1739. John ER, Ahn H, Prichep L. Developmental equations for the electroencephalogram. Science 1980; 210: 1255-8.

John ER, Prichep LS, Fridman J, Easton P. Neurometrics: Computer-assisted differential diagnosis of brain dysfunctions. Science 1988; 239: 162-169. Kaspar F, Schuster HG. Easily calculable measure for the complexity of spatiotemporal patterns. Phys Rev A 1987; 36: 842-848. 
Kochunov P, Williamson DE, Lancaster J, Fox P, Cornell J, Blangero J, et al. Fractional anisotropy of water diffusion in cerebral white matter across the lifespan. Neurobiol Aging 2012; 33:9-20.

Kolmogorov AN. Three approaches to the quantitative definition of information. Inf Trans $1965 ; 1: 3-11$.

Lebel C, Walker L, Leemans A, Phillips L, Beaulieu C. Microstructural maturation of the human brain from childhood to adulthood. Neuroimage 2008; 40: 1044-55. Lempel A, Ziv J. On the complexity of finite sequences. IEEE Trans Inform Theory 1976; $22: 75-81$.

Li Y, Tong S, Liu D, Gai Y, Wang X, Wang J, et al. Abnormal EEG complexity in patients with schizophrenia and depression. Clin Neurophysiol 2008; 119: 1232-41. Lindsley DB. A longitudinal study of the occipital alpha rhythm in normal children: frequency and amplitude standards. J Genetic Psychol 1939; 55: 197-213.

Lippe S, Kovacevic N, McIntosh AR. Differential maturation of brain signal complexity in the human auditory and visual system. Front Hum Neurosci 2009; 3: 1-9.

Lopes da Silva F. Neural mechanisms underlying brain waves: from neural membranes to networks. Electroencephalogr Clin Neurophysiol 1991; 79: 81-93.

Luders E, Narr KL, Thompson PM, Rex DE, Jancke L, Steinmetz H, et al. Gender differences in cortical complexity. Nat Neurosci 2004; 7: 799-800.

LutzenbergerW, Preissl, H, Pulvermuller F. Fractal dimension of electroencephalographic time series and underlying brain processes. Biol Cybern 1995; 73: 477-82.

Marshall PJ, Bar-Haim Y, Fox NA. Development of the EEG from 5 months to 4 years of life. Clin Neurophysiol 2002; 113: 1199-1208. 
Matousek M, Petersén I. Norms for the EEG. In: Kellaway P, Petersén I, editors. Automation of Clinical Electroencephalography. New York: Raven, 1973: 75-102. McGraw P, Liang L, Provenzale JM. Evaluation of normal age-related changes in anisotropy during infancy and childhood as shown by diffusion tensor imaging. AJR Am J Roentgenol 2002; 179: 1515-22.

McIntosh AR, Kovacevic N, Itier RJ. Increased brain signal variability accompanies lower behavioral variability in development. PLoS Comput Biol 2008; 4: e1000106. Mackey MC, Milton JG. Dynamical diseases. Ann N Y Acad Sci 1987; 504: 16-32. McLaughlin NC, Paul RH, Grieve SM, Williams LM, Laidlaw D, DiCarlo M, et al. Diffusion tensor imaging of the corpus callosum: a cross-sectional study across the lifespan. Int J Dev Neurosci 2007; 25: 215-21.

Méndez MA, Zuluaga P, Hornero R, Gómez C, Escudero J, Rodríguez-Palancas A, et al. Complexity analysis of spontaneous brain activity: effects of depression and antidepressant treatment. J Psychopharmacol 2011, doi: 10.1177/0269881111408966. Meyer-Lindenberg A. The evolution of complexity in human brain development: an EEG study. Electroencephalogr Clin Neurophysiol 1996; 99: 405-11.

Misic B, Vakorin VA, Paus T, McIntosh AR. Functional embedding predicts the variability of neural activity. Front Syst Neurosci 2011; 90: 1-6.

Nagarajan R. Quantifying physiological data with Lempel-Ziv complexity - Certain issues. IEEE Trans Biomed Eng 2002; 49: 1371-1373.

Nusbaum AO, Tang CY, Buchsbaum MS, Wei TC, Atlas SW. Regional and global changes in cerebral diffusion with normal aging. AJNR Am J Neuroradiol 2001; 22: $136-42$.

Peters M. Sex differences in human brain size and the general meaning of differences in brain size. Can J Psychol 1991; 45: 507-22. 
Pfefferbaum A, Adalsteinsson E, Sullivan EV. Frontal circuitry degradation marks healthy adult aging: Evidence from diffusion tensor imaging. Neuroimage 2005; 26 : 891-9.

Pfefferbaum A, Mathalon DH, Sullivan EV, Rawles JM, Zipursky RB, Lim KO. A quantitative magnetic resonance imaging study of changes in brain morphology from infancy to late adulthood. Arch Neurol 1994; 51: 874-87.

Pravitha R, Sreenivasan R, Nampoori VP. Complexity analysis of dense array EEG signal reveals sex difference. Int J Neurosci 2005; 115: 445-60.

Radhakrishnan N, Gangadhar BN. Estimating regularity in epileptic seizure time-series data. A complexity-measure approach. IEEE Eng Med Biol 1998; 17: 89-94.

Rossini PM, Rossi S, Babiloni C, Polich J. Clinical neurophysiology of aging brain: from normal aging to neurodegeneration. Prog Neurobiol 2007; 83: 375-400. Segalowitz SJ, Santesso DL, Jetha MK. Electrophysiological changes during adolescence: a review. Brain Cogn 2010; 72: 86-100.

Snook L, Paulson L.A, Roy D, Phillips L, Beaulieu C. Diffusion tensor imaging of neurodevelopment in children and young adults. Neuroimage 2005; 26: 1164-73. Solesio E, Lorenzo-López L, Campo P, López-Frutos JM, Ruiz-Vargas JM, Maestu F. Retroactive interference in normal aging: a magnetoencephalography study. Neurosci Lett $2009 ; 456: 85-8$.

Sowell ER, Peterson BS, Thompson PM, Welcome SE, Henkenius AL, Toga AW. Mapping cortical change across the human life span. Nat Neurosci 2003; 6: 309-15. Sowell ER, Thompson PM, Toga AW. Mapping changes in the human cortex throughout the span of life. Neuroscientist 2004;10: 372-92.

Stam C. Nonlinear dynamical analysis of EEG and MEG: Review of an emerging field. Clin Neurophysiol 2005; 116: 2266-2301. 
Tononi G, Sporns O, Edelman GM. A measure for brain complexity: relating functional segregation and integration in the nervous system. Proc Natl Acad Sci USA 1994; 91: $5033-7$.

Vakorin VA, Misic B, Krakovska O, McIntosh AR. Empirical and theoretical aspects of generation and transfer of information in a neuromagnetic source network. Front Syst Neurosci 2011; 5: 1-6.

Whitford TJ, Rennie CJ, Grieve SM, Clark CR, Gordon E, Williams LM. Brain maturation in adolescence: concurrent changes in neuroanatomy and neurophysiology. Hum Brain Mapp 2007; 28: 228-37.

Witelson SF. Hand and sex differences in the isthmus and genu of the human corpus callosum. A postmortem morphological study. Brain 1989; 112: 799-835.

Witelson SF, Glezer II, Kigar DL. Women have greater density of neurons in posterior temporal cortex. J Neurosci 1995; 15: 3418-28.

Yakovlev PI, Lecours AR. The myelogenetic cycles of regional maturation of the brain In: Minkowski A, editor. Regional Development of the Brain Early in Life. Boston: Blackwell Scientific Publications Inc., 1967: 3-70.

Yan R, Gao RX. Complexity as a measure for machine health evaluation. IEEE Trans Instrum Meas 2004; 53:1327-34.

Zhang XS, Roy RJ, Jensen EW. EEG complexity as a measure of depth of anesthesia for patients. IEEE Trans. Biomed Eng 2001; 48: 1424-33.

Zhang XS, Zhu YS, Thakor NV, Wang ZZ. Detecting ventricular tachycardia and fibrillation by complexity measure. IEEE Trans Biomed Eng 1999; 46: 548-555. 


\section{Table and Figure Legends}

Table 1. Polynomial Regression analyses for Anterior, Central and Posterior regions. In this particular case fittings are displayed by gender, since we found significant differences between males and females in the exploratory ANOVA. The determination coefficients $\left(\mathrm{R}^{2}\right)$ are displayed as a measure of goodness of fit and the $\mathrm{p}$-value for $\mathrm{R}^{2}$ is displayed in the bottom row of each cell on the second column. The significance of these coefficients indicates that the quadratic fit is better than the linear fit in all genders and regions. The age-of -peak (maximum) for the polynomial is given in the last column of the Table.

Table 2. Polynomial Regression analyses for Right lateral and left lateral. As for Table 1 , the $\mathrm{p}$-value for $\mathrm{R}^{2}$ is displayed in the bottom row of each cell on the second column. The significance of these coefficients indicates that the quadratic fit is better than the linear fit in both regions. The age-of -peak (maximum) for the polynomial is given in the last column of the Table

Figure 1. Sensor-space representation of the five regions submitted to statistical analyses.

Figure 2. Mean and standard error values of LZC scores in the five regions, represented according to Age and Gender groups. Males' information is displayed on top, while females information is displayed on the bottom of the figure.

Figure 3. Gender x Age effects in Anterior region (mean and standard error). Females complexity values are represented by dashed lines while males' values are represented by solid lines. 
Figure 4. Data points (female=triangle, male $=$ square) and fitted models of LZC variables represented as a quadratic function of Age. For Anterior, Central and Posterior regions the polynomial regression models were presented separately for males (solid line) and females (dashed line). 
Table 1.

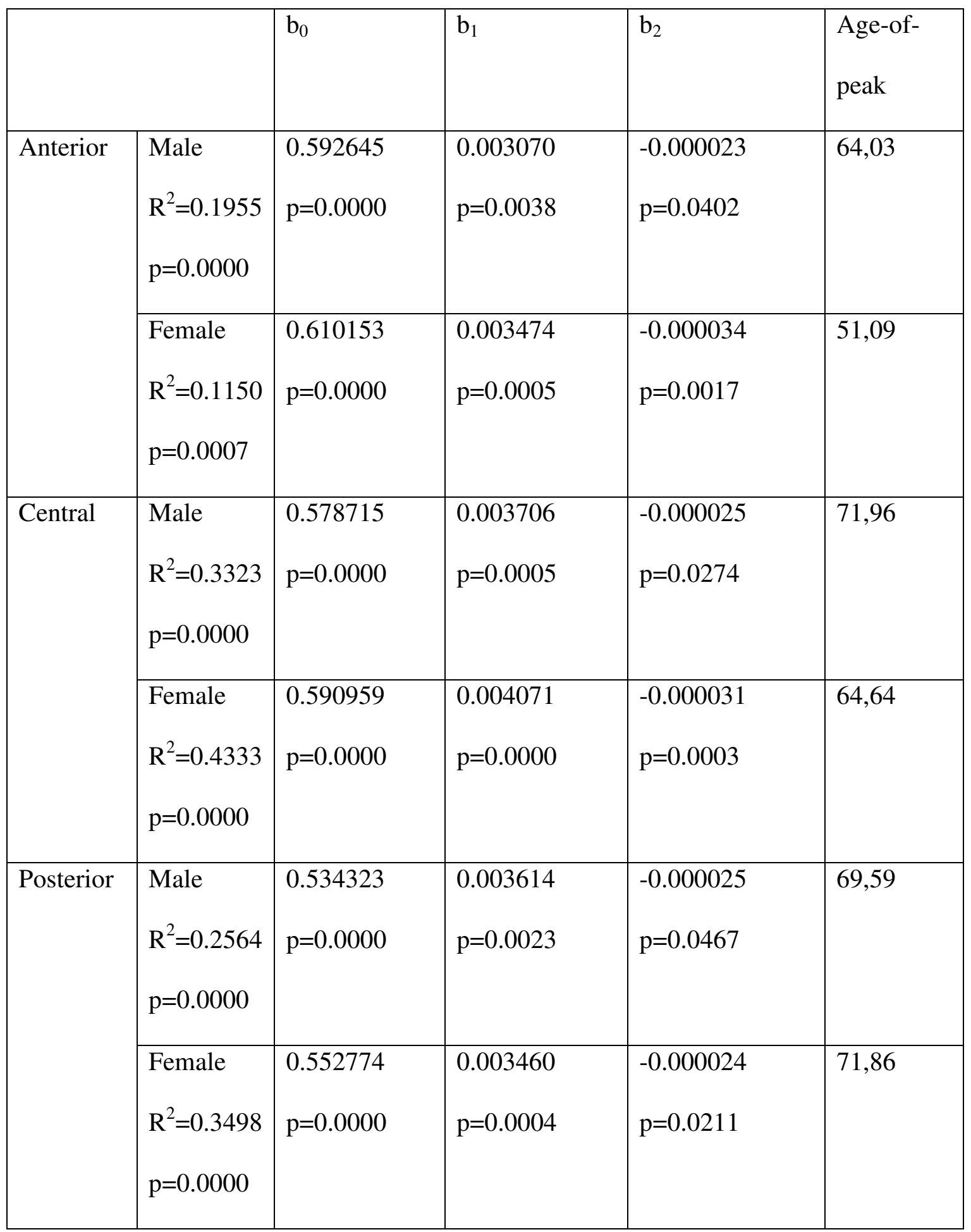


Table 2.

\begin{tabular}{|r|l|l|l|l|}
\hline & $\mathrm{b}_{0}$ & $\mathrm{~b}_{1}$ & $\mathrm{~b}_{2}$ & Age-of- \\
& & & & peak \\
\hline $\mathrm{R}^{2}=0.2777$ & $\mathrm{p}=0.0000$ & $\mathrm{p}=0.0000$ & $\mathrm{p}=0.0001$ & \\
$\mathrm{p}=0.0000$ & & & & \\
\hline Reft_Lateral & & & & \\
$\mathrm{R}{ }^{2}=0.2922$ & $\mathrm{p}=0.0000$ & $\mathrm{p}=0.0000$ & $\mathrm{p}=0.0167$ & \\
$\mathrm{p}=0.0000$ & & & & \\
\hline
\end{tabular}


Figure 1. Sensor-space representation of the five regions submitted to statistical analyses.

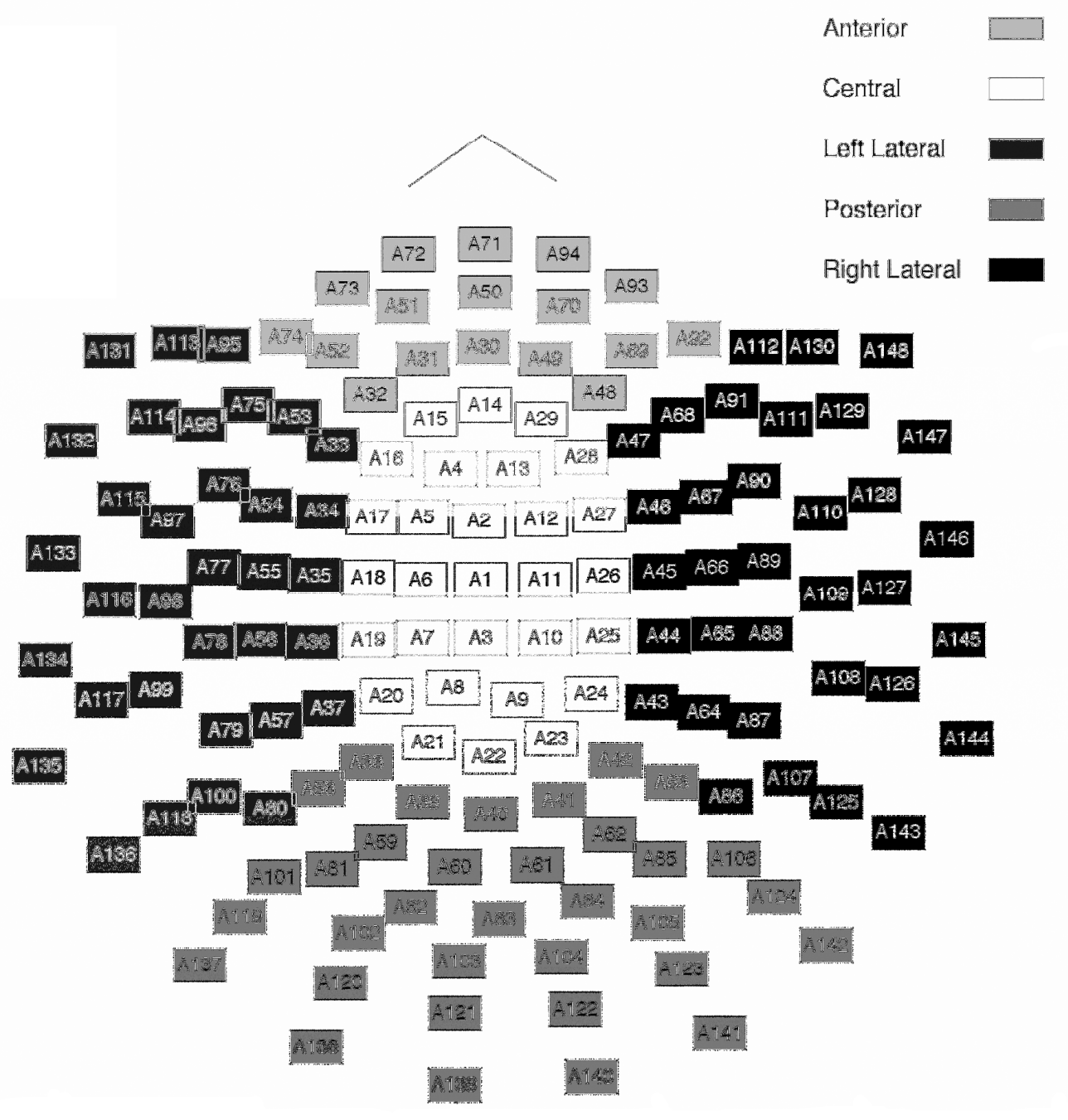


Figure 2. Mean and standard error values of LZC scores in the five regions, represented according to Age and Gender groups. Males' information is displayed on top, while females information is displayed on the bottom of the figure.
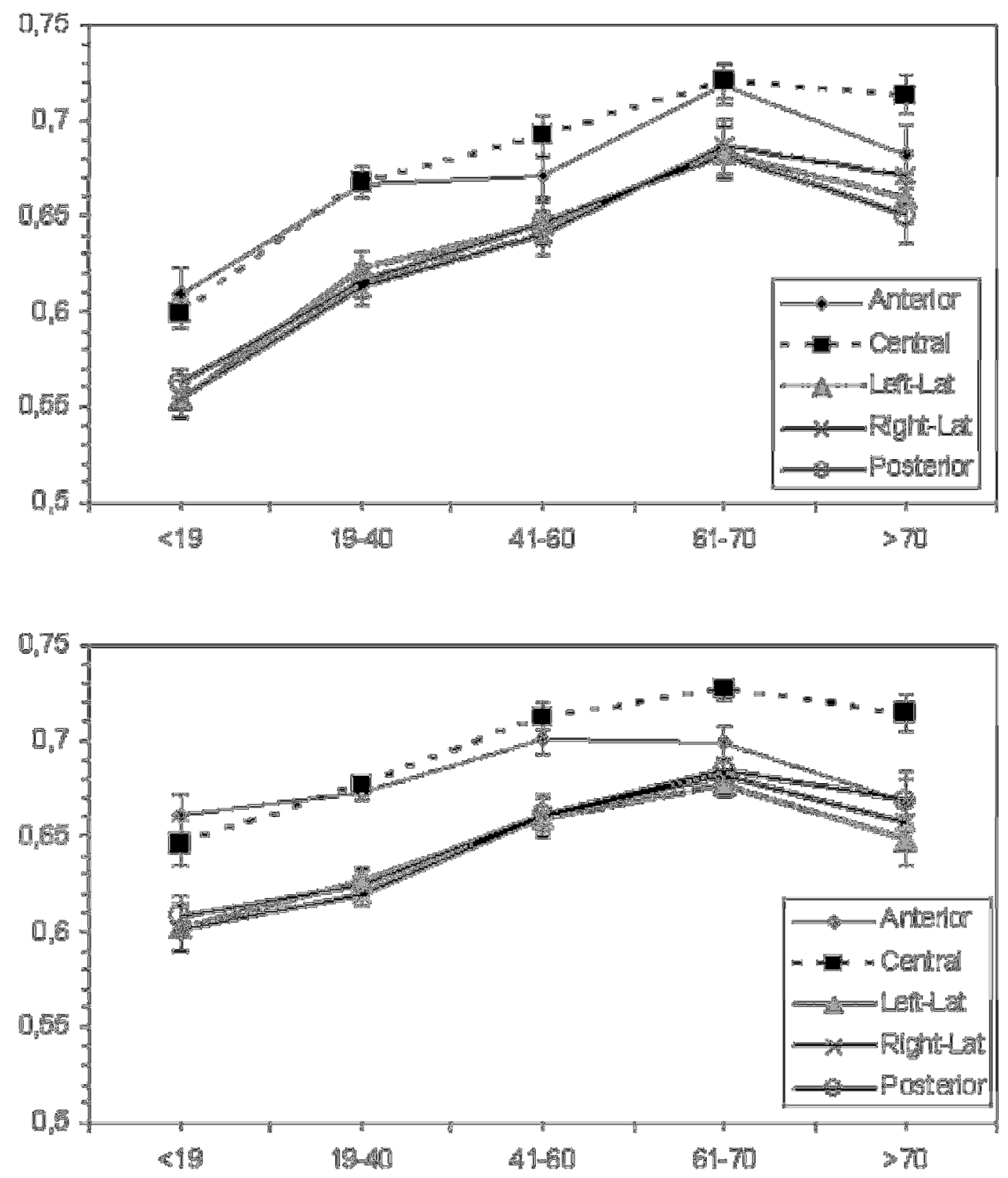
Figure 3. Gender x Age effects in Anterior region (mean and standard error). Females complexity values are represented by dashed lines while males' values are represented by solid lines.

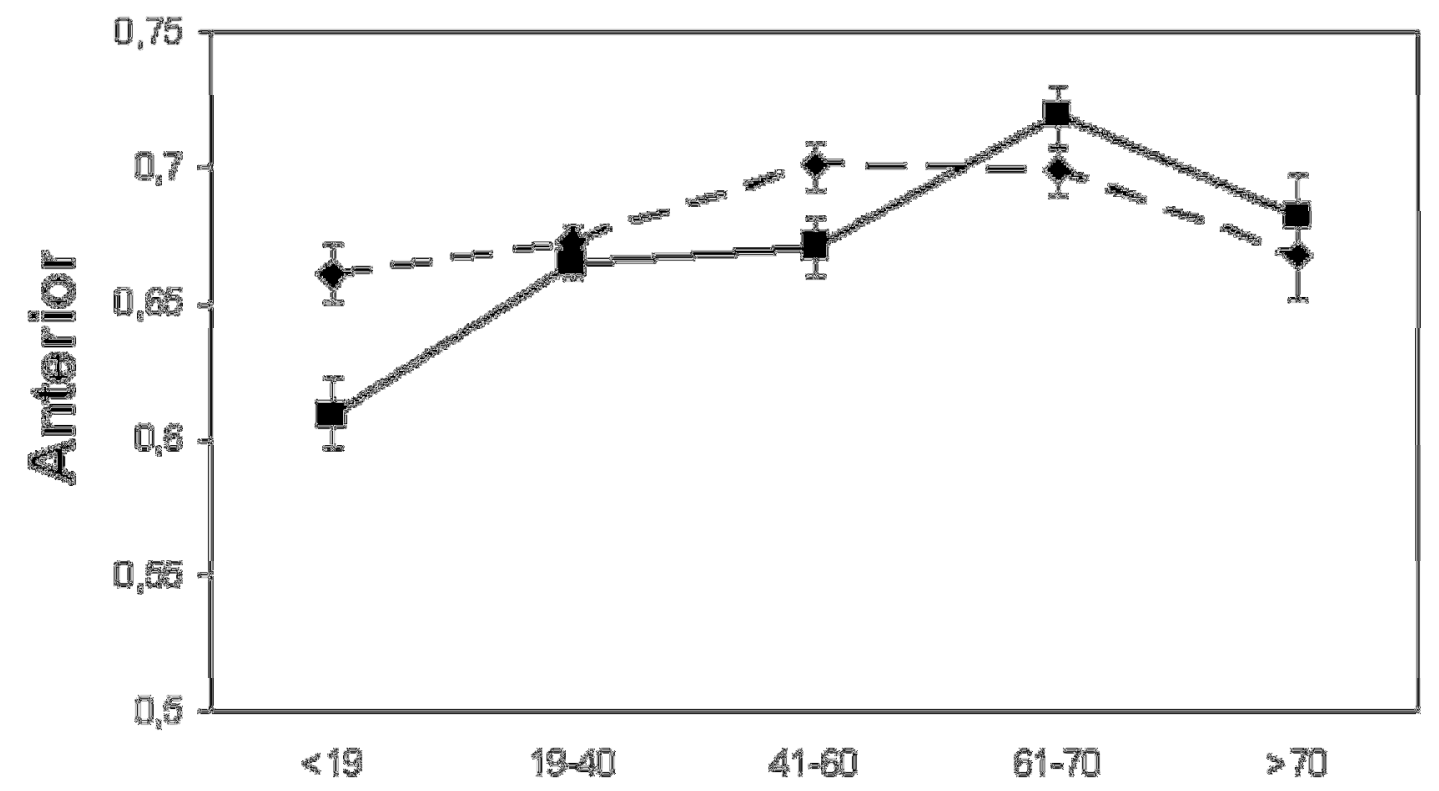


Figure 4. Data points (female=triangle, male $=$ square) and fitted models of LZC variables represented as a quadratic function of Age. For Anterior, Central and Posterior regions the polynomial regression models were presented separately for males (solid line) and females (dashed line).
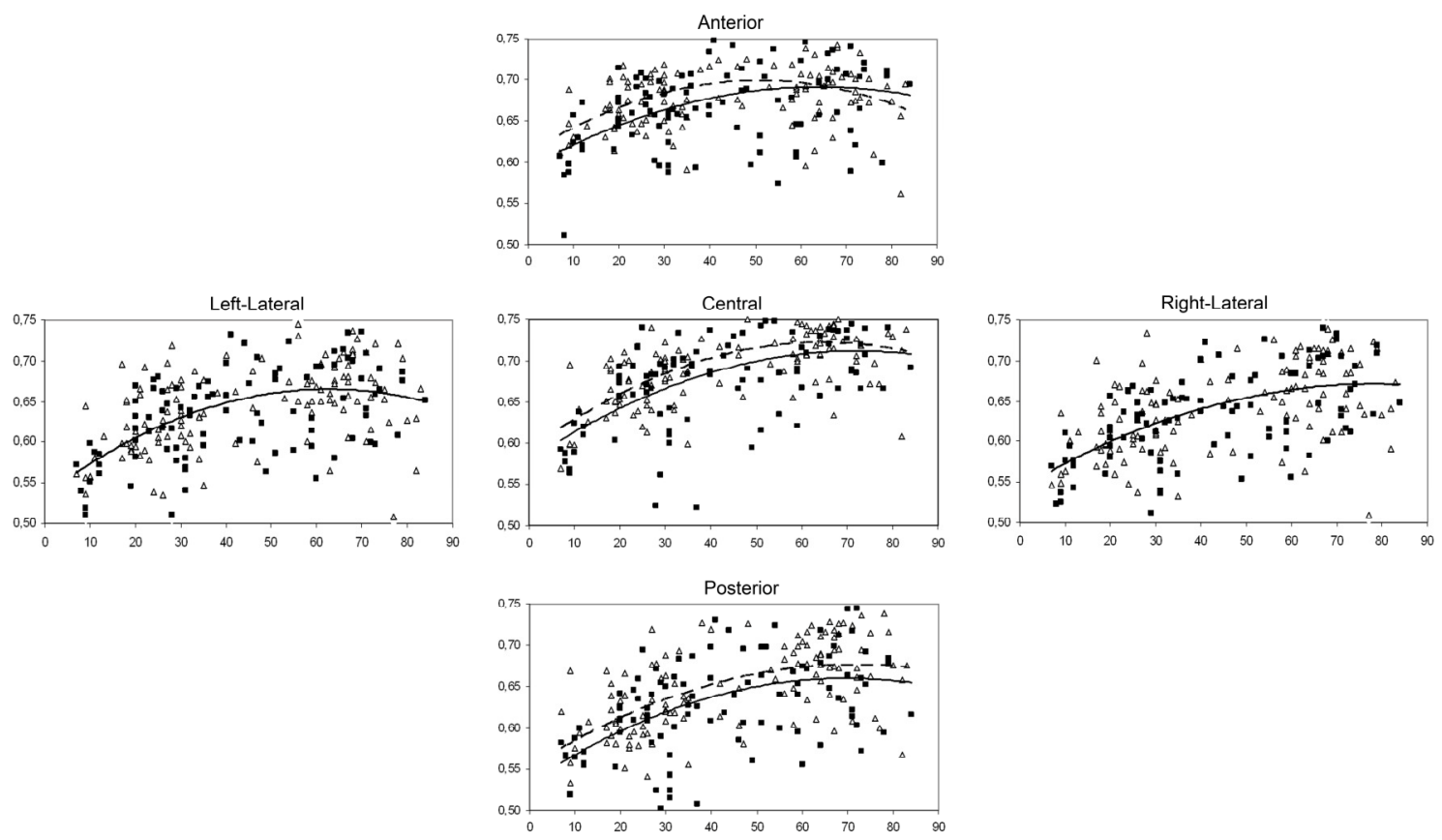